\title{
Normal-tension glaucomatous optic neuropathy is related to blood pressure variability in the Maracaibo Aging Study
}

\author{
Jesus D. Melgarejo ${ }^{1,2}$, Gladys E. Maestre ${ }^{2,3,4}$, Luis J. Mena ${ }^{5}$, Joseph H. Lee ${ }^{6,7}$, Michele \\ Petitto $^{8}$, Carlos A. Chávez ${ }^{2}$, Gustavo Calmon ${ }^{9}$, Egle Silva ${ }^{9}$, Lutgarde Thijs ${ }^{1}$, Lama A. Al- \\ Aswad $^{10}$, Joseph D. Terwilliger ${ }^{11,12,13}$, C. Gustavo De Moraes ${ }^{14}$, Fang-Fei Wei ${ }^{1}$, Thomas \\ Vanassche $^{15}$, Peter Verhamme ${ }^{15}$, Jan A. Staessen ${ }^{1,16,17}$, Zhen-Yu Zhang ${ }^{1}$ \\ ${ }^{1}$ Studies Coordinating Centre, Research Unit Hypertension and Cardiovascular Epidemiology, KU \\ Leuven Department of Cardiovascular Sciences, University of Leuven, Leuven, Belgium \\ ${ }^{2}$ Laboratory of Neurosciences, Faculty of Medicine, University of Zulia, Maracaibo, Zulia, \\ Venezuela \\ ${ }^{3}$ Department of Neurosciences and Department of Human Genetics, University of Texas Rio \\ Grande Valley School of Medicine, Brownsville, TX, USA \\ ${ }^{4}$ Alzheimeŕs Disease Resource Center for Minority Aging Research, University of Texas Rio \\ Grande Valley, Brownsville, TX, USA \\ ${ }^{5}$ Department of Informatics, Universidad Politécnica de Sinaloa, Mazatlán, México \\ ${ }^{6}$ Taub Institute for Research in Alzheimer's Disease and the Aging Brain and G.H. Sergievsky \\ Center at Columbia University Medical Center, New York, USA \\ ${ }^{7}$ Departments of Epidemiology and Neurology, Columbia University Medical Center, New York, \\ NY, USA \\ ${ }^{8}$ Glaucoma and Retina Units, Eye Clinic of Maracaibo, Maracaibo, Zulia, Venezuela \\ ${ }^{9}$ Laboratory of Ambulatory Recordings, Cardiovascular Institute (IECLUZ), University of Zulia, \\ Maracaibo, Zulia, Venezuela \\ ${ }^{10}$ Department of Ophthalmology, New York University (NYU) School of Medicine, NYU Langone \\ Health, New York, NY, USA \\ ${ }^{11}$ Department of Genetics and Development, Department of Psychiatry, and G.H. Sergievsky \\ Center, Columbia University, New York, NY, USA \\ ${ }^{12}$ Division of Medical Genetics, New York State Psychiatric Institute, New York, NY, USA \\ ${ }^{13}$ Division of Public Health Solutions, National Institute for Health and Welfare, Helsinki, Finland \\ ${ }^{14}$ Department of Ophthalmology, Columbia University, New York, NY, USA
}

\footnotetext{
${ }^{凶}$ Zhen-Yu Zhang, zhenyu.zhang@med.kuleuven.be.

Supplementary information The online version contains supplementary material available at https://doi.org/10.1038/ s41440-021-00687-1.

Conflict of interest The authors declare no competing interests.
} 
${ }^{15}$ Center for Molecular and Vascular Biology, KU Leuven Department of Cardiovascular Sciences, University of Leuven, Leuven, Belgium

${ }^{16}$ Cardiovascular Research Institute Maastricht (CARIM), Maastricht University, Maastricht, The Netherlands

${ }^{17}$ Alliance for the Promotion of Preventive Medicine (APPREMED), Mechelen, Belgium

\section{Abstract}

Hypoperfusion of the optic nerve might be involved in the pathogenesis of normal-tension glaucomatous optic neuropathy (GON). Mean arterial pressure (MAP) drives ocular perfusion, but no previous studies have addressed the risk of GON in relation to blood pressure (BP) variability, independent of BP level. In a cross-sectional study, 93 residents of Maracaibo, Venezuela, underwent optical coherence tomography, visual field assessments and 24-h ambulatory BP monitoring between 2011 and 2016. We investigated the association of normal-tension GON with or without visual field defects with reading-to reading variability of 24-h MAP, as captured by variability independent of the MAP level (VIM $\mathrm{VIap}_{\text {ma }}$ ). Odds ratios (ORs) were adjusted for 24-h MAP level and for a propensity score of up to five risk factors. Among the 93 participants $(87.1 \%$ women; mean age, 61.9 years), 26 had open-angle normal-tension GON at both eyes; 14 had visual field defects; and 19 did not have visual field defects. The OR ratios for normal-tension GON, expressed per 1-SD increment in $\operatorname{VIM}_{\text {map }}(2 \mathrm{~mm} \mathrm{Hg}$ ), were 2.17 (95\% confidence interval, 1.33-3.53) unadjusted; 2.20 (1.35-3.61) adjusted for 24-h MAP level only; 1.93 (1.10-3.41) with additional adjustment for age, educational attainment, high-density lipoprotein (HDL) cholesterol and office hypertension; and 1.95 (1.10-3.45) in models including intraocular pressure. We confirmed our a priori hypothesis that BP variability, most likely operating via hypoperfusion of the optic nerve, is associated with normal-tension GON. 24-H ambulatory BP monitoring might therefore help stratify the risk of normal-tension GON.

\section{Keywords}

ambulatory blood pressure monitoring; blood pressure variability; normal-tension glaucoma visual; visual field defects; population science

\section{Introduction}

Vascular dysregulation might play a role in the pathogenesis of glaucomatous optic neuropathy (GON), particularly in the presence of normal intraocular pressure [1]. The mean ocular perfusion pressure is the difference between the pressure in the central retinal artery and vein [2]. The central retinal arterial perfusion pressure can be determined from the mean arterial pressure (MAP) in the brachial artery and the intraocular pressure [2]. Fluctuations in mean ocular perfusion pressure can lead to unstable ocular blood flow [1], thereby compromising the oxygen supply to the optic nerve head [3]. This results in the interruption of axonal transport and subsequent death of ganglion cells in the inner nuclear layer of the retina and creates a toxic micro-environment leading to further secondary retinal ganglion cell loss [4]. 
According to current guidelines, ambulatory blood pressure (BP) monitoring is the state-ofthe-art technology to estimate a person's BP and to quantify diurnal changes in BP [5]. In a previous publication [6], we demonstrated that a lower nighttime-to-daytime BP ratio was associated with a higher risk of GON. Building on these preliminary findings, we hypothesized that normal-tension GON might be associated with reading-to-reading BP variability, as assessed by 24-h ambulatory BP monitoring. We tested this hypothesis in a cross-sectional analysis of 93 people enrolled in the Maracaibo Aging Study [7], of whom 26 had GON [6].

\section{Methods}

\section{Study population}

The Maracaibo Aging Study is a population-based study of chronic age-related diseases that enrolled 2968 participants from the Santa Lucia (1998) and Santa Rosa de Agua (2010) neighborhoods of Maracaibo, Venezuela (Table S1, Supplementary file) [7]. The initial participation rate was 70.0\%. From August 2011 until June 2016, we recruited an additional 534 participants using a sampling frame prioritizing the female ancestral line according to recorded pedigrees with the intention of engaging in genetic studies (NIH project number, 5R01AG036469-02). We randomly selected 130 participants older than 40 years and offered them an in-depth eye examination; 114 accepted the invitation (participation rate, 87.7\%). The Institutional Review Boards of the Cardiovascular Institute at the University of Zulia, Maracaibo and Columbia University, New York, approved the study, which complied with the Declaration of Helsinki [8]. All participants renewed informed written consent. We excluded 21 participants from the analysis because they declined 24-h ambulatory BP monitoring $(n=20)$ or because the intraocular pressure was higher than $22 \mathrm{mmHg}$. Thus, 93 participants were statistically analyzed, of whom 26 (28.0\%) had normal-tension GON.

\section{Ophthalmological assessment}

An ophthalmologist with experience in glaucomatous eye disease (Petitto) performed the examination of both eyes in Maracaibo. The examination included history taking, measurement of visual acuity with correction, slit lamp examination, and a mydriatic assessment of the lens, vitreous body, and retina. Intraocular pressure was estimated by Goldmann applanation tonometry. The optic nerve head and the thickness of the retinal fiber layer were evaluated by spectral domain optic coherence tomography (Spectralis ${ }^{\circledR}$, Heidelberg Engineering, GmbH, Heidelberg, Germany) interfaced with a computer running Spectralis ${ }^{\circledR}$ software, version 5.4.7.0. The visual field was assessed using the Heidelberg Edge Perimeter ${ }^{\circledR}$ (Heidelberg Engineering GmbH, Heidelberg, Germany). An abnormal optic disk was diffuse or focal narrowing or notching of the optic disc rim, or optic disc neural rim asymmetry of the two eyes consistent with loss of neural tissue. An abnormal visual field was visual field loss consistent with retinal nerve fiber layer damage (e.g., nasal step, arcuate field defect, or paracentral depression in clusters of test sites) based on the presence of abnormal clusters or visual field defects consistent with glaucomatous optic nerve damage. All ocular tomographic images and visual field results were read a second time by an independent specialist at Columbia University (De Moraes), resulting in -coefficients $\searrow 0.90$, indicating high interreader reproducibility [9]. 
GON with visual field defects was diagnosed during the optic clinical disc evaluation and was confirmed by measurement of retinal nerve fiber layer thickness by spectral domain optic coherence tomography. GON without visual field defects was diagnosed if the patient met the criteria for GON but not those for visual field defects. Gonioscopy confirmed that all patients were open-angle cases. There were no cases with secondary glaucomatous neuropathy.

\section{Ambulatory BP monitoring}

Validated [10] oscillometric 90207 Spacelabs monitors (Snoqualmie, WA) were programmed to obtain readings at 15 -min intervals from 6 a.m. until 11 p.m. and at 30-min intervals from 11 p.m. until 6 a.m. We limited our analyses of the ambulatory recordings to MAP because of its known relation to mean ocular perfusion pressure [2] and because oscillometric devices measure MAP and extrapolate systolic and diastolic BP [11]. The same SAS macro processed all ambulatory recordings, which stayed unedited with the exception of readings with higher diastolic than systolic BP or flagged with an error code. The within-subject 24-h MAP was a time-weighted average, giving weights to each individual reading proportional to the preceding time interval.

\section{Statistical analysis}

For database management and statistical analysis, we used SAS, version 9.4 (SAS Institute, Cary, NC). The 24-h variability of MAP was determined from the weighted SD, the coefficient of variation, the difference between the maximum and minimum MAP level during the recording, average real variability [12], and variability independent of the mean (VIM) [13]. Average real variability is the mean of the absolute differences between consecutive BP measurements [12]. VIM is calculated as the SD divided by the mean to the power $\mathrm{x}$ and multiplied by the population mean to the power $\mathrm{x}$ [13]. The power $\mathrm{x}$ is obtained by fitting a curve through a plot of SD against mean, using the model $\mathrm{SD}=\mathrm{a} \times$ meanx, where $\mathrm{x}$ was derived by nonlinear regression analysis. We assessed associations between the level and variability of MAP using Pearson correlation coefficients. We compared means by ANOVA or $t$-tests, as appropriate, and proportions by Fisher's exact test. The probability of GON associated with the level and variability of MAP was computed by logistic regression analyses in unadjusted and adjusted models. Multivariable-adjusted models included the level and variability of MAP and a propensity score of BP variability. The propensity score, computed in view of the relatively low number of events [14, 15], was derived by regressing BP variability on age, years of education, high-density lipoprotein (HDL) cholesterol and inoffice hypertension, defined according to the 2017 American [5] guidelines. In a sensitivity analysis, we also added intraocular pressure to the propensity score. Significance was a two-tailed a-level of $₫ 0.05$.

\section{Results}

\section{Clinical characteristics}

Table 1 summarizes the characteristics of the study participants. Among the 93 participants, 26 had open-angle GON at or both eyes; 14 had visual field defects, and 19 did not. All patients had intraocular pressure within the normal range (Fig. 1). Intraocular pressure, 
24-h MAP, and VIM of 24-h MAP were normally distributed (Fig. 1). The median number of readings in the 24-h ambulatory BP recordings was 70, 50, and 21 over $24 \mathrm{~h}$, day and nighttime, respectively (Table S2, Supplementary file). There was no difference in the prevalence of GON across thirds of the distribution of intraocular pressure ( $<11 \mathrm{vs.}$ $\geq 11$ to $<14$ vs. $\geq 14 \mathrm{mmHg} ; \mathrm{N}^{\circ}[\%], 5$ [16.7\%] vs. 9 [31.0\%] vs. $5(18.5 \% ; P=0.36)$. The correlation coefficient between intraocular pressure and 24-h MAP was $-0.010(P=$ $0.89)$. In the whole study population, $4(4.3 \%)$ participants were taking diuretics, $9(9.7 \%)$ $\beta$-blockers, 11 (11.8\%) calcium channel blockers, and 31 (33.3\%) angiotensin-converting enzyme inhibitors. These antihypertensive agents were given as monotherapy in 36 patients (38.7\%) or as combination therapy in $10(10.8 \%)$. Intraocular pressure, MAP, and VIM were similar ( $0.09 \geq P \geq 0.96$ ) across groups treated with different drug classes, between those on mono vs. combination therapy, and between participants on or not on antihypertensive drug treatment (Table S3, Supplementary file). Only one participant was receiving treatment to lower the intraocular pressure.

\section{Correlation matrix of intraocular pressure, BP levels, and variability}

The correlation coefficients of the level of 24-h MAP with the variability indexes, including the weighted SD, the coefficient of variation, the difference between the highest and lowest level of 24-h MAP and average real variability, ranged from $0.212(P=0.04)$ for the weighted SD to $0.349(P<0.001)$ for average real variability (Table S4, Supplementary file). Only VIM was not associated with the 24-h MAP (VIMmap; $r=-0.010 ; P=0.96$ ) and was therefore carried out for further analysis.

\section{Association of BP variability with GON}

Unadjusted analysis-In unadjusted analyses across thirds of the VIM distribution (Table 2), the prevalence of GON increased ( $<8.92$ vs. $>8.92$ to $<10.35$ vs. $\geq 10.35 \mathrm{mmHg}$; $\mathrm{N}^{\circ}[\%], 4$ [12.9\%] vs. 8 [25.8\%] vs. $14(45.2 \% ; P=0.02)$. The findings were similar for the prevalence of GON with $(P=0.02)$ and without $(P=0.03)$ visual field defects. The prevalence of office hypertension ( $\geq 130 / \geq 80 \mathrm{mmHg}$ or using antihypertensive drug treatment), age and HDL serum cholesterol increased across thirds of the VIM distribution from 80.7 to $96.8 \%(P=0.04)$, from 60.5 to 67.0 years $(P=0.02)$, and from 42.7 to 48.4 $\mathrm{mg} / \mathrm{dL}(P=0.04)$, respectively (Table 2$)$. None of the other participants' characteristics differed across the VIM distribution $(P \geq 0.08$; Table 2$)$.

Adjusted analysis-Considering all 26 optic neuropathy cases, the odds ratios relating the risk of GON were $2.17(P=0.002)$ for each 1-SD increment in VIMmap $(2 \mathrm{mmHg})$ unadjusted, $2.20(P=0.002)$ when adjusted for the 24-h MAP only, and $1.93(P=0.02)$ with additional adjustment for the propensity score (Fig. 2). The findings were similar when cases with or without visual field detects were analyzed separately (Fig. 2). In sensitivity analyses, including all cases but additionally adjusted for intraocular pressure, the odds ratio for GON was 1.92 (CI, 1.10 to $3.45 ; P=0.02$ ). Further-more, if we replaced the propensity score with the covariables making up the score, the odds ratio was 2.02 (95\% confidence interval, $1.10-3.71 ; P=0.02)$. 


\section{Discussion}

The key finding of our study was that the risk of normal-tension GON increased with higher reading-to-reading VIM of the 24-h MAP and that this association was independent of the 24-h MAP level and other confounders summarized in a propensity score. A sensitivity analysis accounting for intraocular pressure was confirmatory. We chose to analyze 24-h MAP because of the known association of ocular perfusion pressure with brachial MAP. The absence of any association between VIM and the level of the 24-h MAP justified the use of VIMmap as a measure of BP variability.

The few studies [16-19] reporting on the association between normal-tension GON and $\mathrm{BP}$ variability are difficult to interpret. Limitations include a case-control design with unbalanced matching of the number of cases/controls (ranging from 51/28 [16] to 64/82 [18]), a retrospective enrollment of participants [17], evaluation of a randomly selected single eye in each participant [17], a statistical analysis by eyes rather than by people [17], the derivation of beat-to-beat BP variability over a short 30-min period with calibration using a single brachial BP reading and an upper-arm to finger transfer function [17]. One study selectively recruited patients with intraocular pressure below $12 \mathrm{mmHg}$ [19], and another enrolled only patients with GON [17]. In fact, none of the aforementioned studies was population based [16-19]. Two studies [16, 17] computed MAP as diastolic BP plus one-third of pulse pressure, whereas the recommendation was to add $40 \%$ pulse pressure to diastolic BP [20].

To elucidate the role of BP variability in relation to adverse health outcomes, the level of BP needs to be taken into account, as previously demonstrated in several studies. [21-24] Otherwise, the association of BP variability with normal-tension GON is very likely driven by the BP level. Three studies that applied ambulatory BP monitoring used the maximumminimum BP difference $[17,19]$ or the SD $[16,17]$ as measures of BP variability, which in our hands were highly correlated with the BP level with $r$ values greater than 0.212 (Table S4, Supplementary file). No study has assessed the association between normal-tension glaucomatous neuropathy and BP variability accounting for the BP level [16-19]. Our findings demonstrated that BP variability was significantly associated with normal-tension glaucoma independent of the BP level.

Ocular perfusion pressure depends on the intraocular and MAPs. MAP drives the blood flow that maintains the integrity of tissues [25]. Abrupt and continuous variation in MAP can lead to unstable ocular blood flow and supply that lead to the development and progression of glaucomatous damage [1]. Moreover, excessive MAP variability might be ineffectively coped with by eyes with normal-tension glaucoma, as their autoregulatory mechanisms to maintain constant ocular blood flow are impaired [3]. This principle is also observed in the brain, as in the presence of poor cerebral autoregulation to main blood flow, the excessive variation in the BP levels increased the risk of poor outcomes [26].

In our study participants, both eyes were assessed. We did not compute but measured the MAP, which determines the ocular perfusion pressure. We used VIMmap, a BP variability index that is unrelated to the BP level $(r=-0.012)$. All ocular examinations included 
spectral domain optical coherence tomography and an evaluation of the visual field. Two independent observers assessed the images with intergrader $\kappa$-statistics of $90 \%$ or higher. Other causes of optic neuropathy, such as congenital, compressive, toxic, or traumatic neuropathy, were excluded from the ophthalmological assessment. In view of the relatively small number of events in our dataset $[14,15]$, we reduced the number of covariables in the multivariable-adjusted logistic regression by summarizing potential confounders in a propensity score. However, replacing the propensity score with the actual covariables did not affect our results, probably because proportional to the total sample size, cases had a high (28.0\%) prevalence. This rate of normal-tension glaucoma is higher than that observed among Black Hispanics living in Barbados (4.4\%) 21 or among Mexican Americans living in Los Angeles (4.7\%) [27] but was similar to the prevalence of suspected glaucoma among Hispanics of Caribbean origin residing in New York (24.9\%) [28].

Notwithstanding the strong points of our current study, it must also be interpreted within the context of its limitations. First, women were overrepresented due to the sampling frame favoring the female ancestral line. Inhabitants of Maracaibo have a mixed European and native Venezuelan ancestry. Our findings can therefore not be easily extrapolated to men or other ethnic groups. Second, we did not measure the diurnal variation in intraocular pressure. However, there was no correlation between the single intraocular pressure measurement performed during the day and the daytime MAP $(r=0.032 ; P=0.60)$. Finally, the cross-sectional design does not allow causal inferences to be made, although our observations do confirm a plausible pathophysiological mechanism of age-related normaltension GON proposed by other investigators [29].

In conclusion, the risk of normal-tension GON increased almost twofold for each 2-mmHg increment in VIMmap, of which the 5th to 95th percentile interval ranged from 6.5 to $13.9 \mathrm{mmHg}$ (Fig. 1). BP variability oscillates around the mean BP level during the whole day. The clinical implications of our findings should be viewed within the context of the 2017 American guidelines for the management of hypertension, which proposes BP-lowering treatment when patients with stage- 1 hypertension stay uncontrolled with lifestyle intervention alone [5]. If the BP is lowered excessively, patients become vulnerable to glaucomatous damage to the optic nerve, especially at night [6], when the ocular perfusion pressure might fall to too low levels, resulting in ischemic damage to the optic disk. Ophthalmologists should therefore embrace 24-h ambulatory BP as part of their diagnostic armamentarium to stratify for risk for glaucomatous eye disease, particularly in patients on antihypertensive drug treatment [29]. To facilitate this approach, manufacturers might incorporate an algorithm in their software computing VIMmap from individual patient readings and country-, sex-, and age-specific population means. Such information is available in the International Database on Ambulatory BP in Relation to Cardiovascular Outcomes [30, 31].

\section{Supplementary Material}

Refer to Web version on PubMed Central for supplementary material. 


\section{Acknowledgements}

The authors thank the participants and assessment team of the MAS in both Santa Lucía and Santa Rosa. We also acknowledge the fellows Drs. Federico Flores, Rubén Torre Alba, Roxana Chacón, and David Santana, who collected ophthalmological information and support from Dr. Doris Molina. We also acknowledge the South Texas Diabetes and Obesity Institute for support in preparation of this report. The report is supported by the GeneEnvironment Interaction in Cognition in Venezuela Families project founded by the National Institute on AgingNational Institutes of Health under Award Number R01AG036469 (G.E.M. and J.D.T.) and 1 R21EY029605-01 (G.E.M. and L.A. A.-A.).

\section{References}

1. Heijl A, Leske MC, Bengtsson B, Hyman L, Bengtsson B, Hussein M. Reduction of intraocular pressure and glaucoma progression: results from the early manifest glaucoma trial. Arch Ophthalmol. 2002;120:1268-1279. [PubMed: 12365904]

2. Van Keer K, Breda JB, Pinto LA, Stalmans I, Vandewalle E. Estimating mean ocular perfusion pressure using mean arterial pressure and intraocular pressure. Invest Ophthalmol Vis Sci. 2016;57:2260. [PubMed: 27124318]

3. Flammer J, Orgül S, Costa VP, Orzalesi N, Krieglstein GK. Serra LMet al.The impact of ocular blood flow in glaucoma. Prog Retin Eye Res. 2002;21:359-393. [PubMed: 12150988]

4. Jonas JB, Aung T, Bourne RR, Bron AM, Ritch R, Panda-Jonas S. Glaucoma. Lancet2017;390:2183-2193. [PubMed: 28577860]

5. Whelton PK, Carey RM, Aronow WS, Casey DE Jr, Collins KJ, Dennison Himmelfarb C, et al.2017 ACC/AHA/AAPA/ABC/ACPM/AGS/APhA/ASH/ASPC/NMA/PCNA guideline for prevention, detection, evaluation, and management of high blood pressure in adults: a report of the American College of Cardiology/American Heart Association Task Force on Clinical Practice Guidelines. J Am Coll Cardiol. 2018;71:e127-e248. [PubMed: 29146535]

6. Melgarejo JD, Lee JH, Petitto M, Yépez JB, Murati FA, Jin Z, et al.Glaucomatous optic neuropathy associated with nocturnal dip in blood pressure. Ophthalmology2017;125:807-814.

7. Maestre GE, Pino-Ramírez G, Molero AE, Silva ER, Zambrano R. Falque Let al.The Maracaibo aging study: population and methodological issues. Neuroepidemiology2002;21:194201. [PubMed: 12065882]

8. World Medical Association. World Medical Association Declaration of Helsinki: ethical principles for medical research invovling human subjects. JAMA2013;310:2191-2194. [PubMed: 24141714]

9. Siegel S, Castellan NJJ. Nominally scaled data and the kappa statistic k. Non-parametric statistics for the behavioural sciences. 1988;2:284-291.

10. Groppelli A, Omboni S, Parati G, Mancia G. Evaluation of non-invasive blood pressure monitoring devices Spacelabs 90202 and 90207 versus resting and ambulatory 24-hour intra-arterial blood pressure. Hypertension1992;20:227-232. [PubMed: 1639465]

11. Ogedegbe GPickering TPrinciples and techniques of blood pressure measurement. Cardiol Clin. 2010;28:571-586. [PubMed: 20937442]

12. Mena L, Pintos S, Queipo NV, Aizpúrua JA, Maestre G, Sulbarán T. A reliable index for the prognostic significance of blood pressure variability. J Hypertens. 2005;23:505-511. [PubMed: 15716690]

13. Rothwell PM, Howard SC, Dolan E, O’Brien E, Dobson JE. Dahlöf Bet al.Prognostic significance of visit-to-visit variability, maximum systolic blood pressure, and episodic hypertension. Lancet. 2010;375:895-905. [PubMed: 20226988]

14. Cepeda MS, Boston R, Farrar JT, Strom BL. Comparison of logistic regression versus propensity score when the number of events is low and there are multiple confounders. Am J Epidemiol. 2003;158:280-287. [PubMed: 12882951]

15. Vittinghoff EMcCulloch CE. Relaxing the rule of ten events per variable in logistic and Cox regression. Am J Epidemiol. 2007;165:710-718. [PubMed: 17182981]

16. Plange N, Kaup M, Daneljan L, Predel H, Remky A, Arend O. 24-h blood pressure monitoring in normal tension glaucoma: nighttime blood pressure variability. J Hum Hypertens. 2006;20:137142. [PubMed: 16239898] 
17. Lee J, Choi J, Jeong D, Kim S, Kook MS. Relationship between daytime variability of blood pressure or ocular perfusion pressure and glaucomatous visual field progression. Am J Ophtalmol. 2015;160:522-537.

18. Lindemann F, Kuerten D, Koch E, Fuest M, Fischer C, Voss A, et al.Blood pressure and heart rate variability in primary open-angle glaucoma and normal tension glaucoma. Curr Eye Res. 2018;43:1507-1513. [PubMed: 30110187]

19. Baek SU, Ha A, Jeoung JW, Park KH, Kim YK. Risk factors for disease progression in low-teens normal-tension glaucoma. Br J Ophthalmol. 2019;4: 10.1136/bjophthalmol-2018-313375.

20. Bos WJ, Verrij E, Vincent HH, Westerhof BE, Parati G, van Montfrans GA. How to assess mean blood pressure properly at the brachial artery level. J Hypertens. 2007;25:751-755. [PubMed: 17351365]

21. Hansen TW, Thijs L, Li Y, Boggia J, Kikuya M. Björklund-Bodegård Ket al.Prognostic value of reading-to-reading blood pressure variability over $24 \mathrm{~h}$ in 8938 subjects from 11 populations. Hypertension2010;55:1049-1057. [PubMed: 20212270]

22. Mancia G, Parati G, Henning M, Flatau B, Omboni S, Glavina F, et al.Relation between blood pressure variability and carotid artery damage in hypertension: baseline data from the European Lacidipine Study on Atherosclerosis (ELSA). J Hypertens. 2001;19:1981-1989. [PubMed: 11677363]

23. Muntner P, Shimbo D, Tonelli M, Reynolds K, Arnett DK, Oparil S. The relationship between visit-to-visit variability in systolic blood pressure and all-cause mortality in general population. Findings from NHANES III, 1988 to 1994. Hypertension. 2011;57:160-166. [PubMed: 21200000]

24. Xu J, Liu Y, Wang A, Gao Y, Wang Y, Wang Y. Blood pressure fluctuation pattern and stroke outcomes in acute ischemic stroke. Hypertens Res. 2019;42:1776-1782. [PubMed: 31451721]

25. DeMers D, Wachs D. Physiology, mean arterial pressure. 2020.

26. Yang M, Pan X, Liang Z, Huang X, Duan M. Cai Het al.Association between blood pressure variability and the short-term outcome in patients with acute spontaneous subarachnoid hemorrhage. Hypertens Res. 2019;42:1701-1707. [PubMed: 31171841]

27. Varma R, Ying-Lai M, Francis BA, Nguyen BBT, Deneen J, Wilson MR, et al.Prevalence of open-angle glaucoma and ocular hypertension in Latinos: the Los Angeles Latino Eye Study. Ophthalmology. 1-8-.2004;111:1439-1448. [PubMed: 15288969]

28. Al-Aswad LA, Joiner DB, Wang X, De Moraes CG, Popplewell D. Amaro-Quireza MLet al. Screening for glaucoma in populations at high risk: the eye screening New York project. Cogent Med. 2017;4:1367059.

29. Leeman MK, Estelyn P. Glaucoma and blood pressure. Hypertension. 2019;73:944-950. [PubMed: 30852921]

30. Yang WY, Melgarejo JD, Thijs L, Zhang ZY, Boggia J. Wei FFet al.Association of office and ambulatory blood pressure with mortality and cardiovascular outcomes. JAMA2019;322:409-420. [PubMed: 31386134]

31. Thijs L, Hansen TW, Kikuya M, Björklund-Bodegård K, Li Y. Dolan Eet al.The International Database of Ambulatory blood pressure in relation to cardiovascular outcome (IDACO): protocol and research perspectives. Blood Press Monit. 2007;12:255-262. [PubMed: 17760218] 

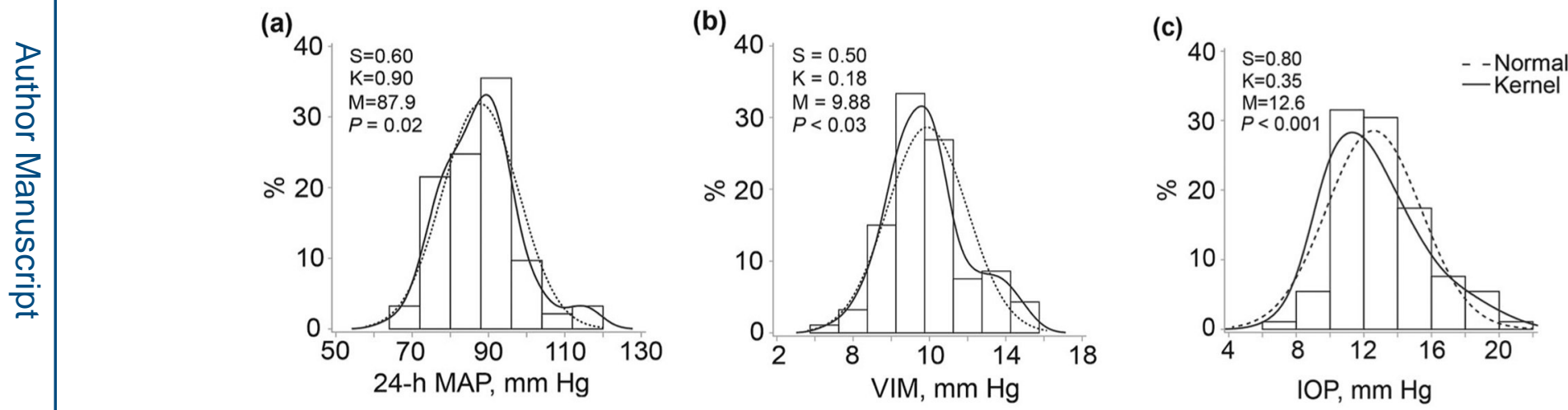

Fig. 1.

Distribution of the level (a) and variability (b) of 24-h mean arterial pressure and of intraocular pressure (c). S and K are the coefficients of skewness and kurtosis. The dotted and full lines represent the normal and kernel density distributions. $M$ is the mean of the distribution. The $P$ values are for departure of the actually observed distribution from normality according to Shapiro-Wilk's statistic. MAP mean arterial pressure, VIM variation independent of the mean MAP level, IOP intraocular pressure 

(a) Unadjusted $\mathrm{Nc} / \mathrm{Nr}$
$P$ value
GON VFD (-) $\quad 19 / 67$

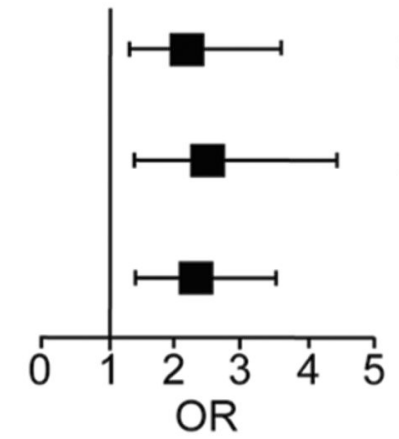
$2.03(1.17-3.51) \quad 0.012$
GON/VFD (+)
$14 / 67$
GON/VFD (+/-)
$26 / 67$
$2.30(1.24-4.30) \quad 0.009$
$2.17(1.33-3.53) \quad 0.002$

(b) Adjusted

GON VFD (-)

GON/VFD (+)

GON/VFD (+/-)

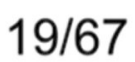

$14 / 67$

$26 / 67$

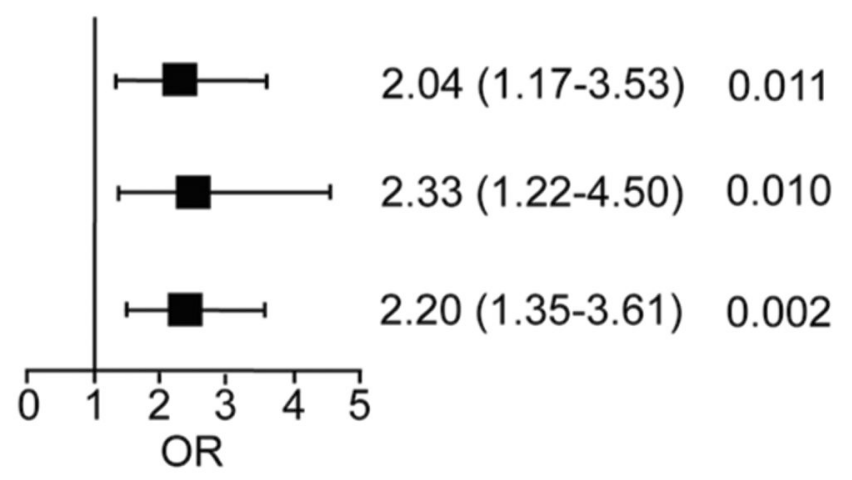

(c)Fully-adjusted

GON VFD (-) 19/67

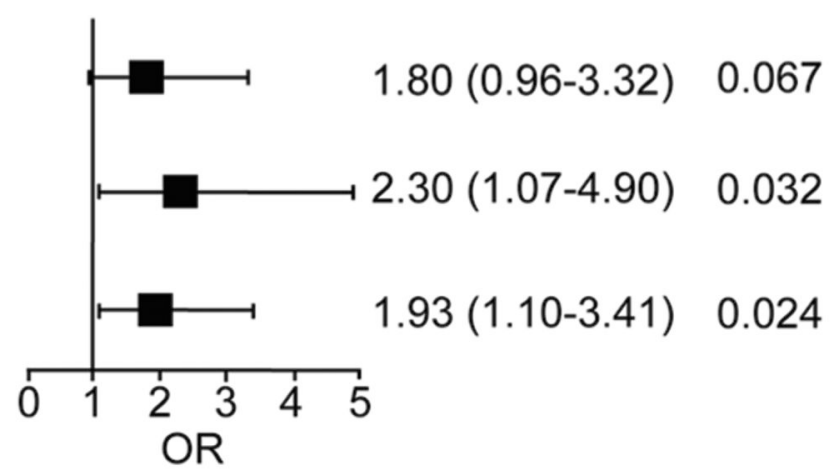

Fig. 2.

Association between glaucomatous optic neuropathy and variability of mean arterial pressure. The risk was expressed per 1 SD on VIM (2 mmHg). Models were unadjusted (a), adjusted for a propensity score derived from age, educational attainment, serum high-density lipoprotein cholesterol, and in-office hypertension (b), and a propensity score additionally accounting for blood pressure level (c). Nc number of cases, $\mathrm{Nr}$ number of participants with healthy eyes, OR odds ratio, CI confidence interval, GON glaucomatous optic neuropathy, VFD visual field defects, VIM variation of mean arterial pressure independent of its mean level, MAP mean arterial pressure 


\section{Table 1}

\section{Characteristics of participants}

\begin{tabular}{|c|c|}
\hline Characteristic & Mean $\pm \mathrm{SD}^{a}{ }_{n=93}$ \\
\hline Age, years & $61.9 \pm 12.9$ \\
\hline Sex, women, $n(\%)$ & $81(87.1 \%)$ \\
\hline Educational attainment (IQR), $\mathrm{y}^{b}$ & $7.6(4.0-11.0)$ \\
\hline Current smoking, $n(\%)^{c}$ & $26(28.0 \%)$ \\
\hline Drinking alcohol, $n(\%)^{c}$ & $9(9.7 \%)$ \\
\hline Body mass index, $\mathrm{kg} / \mathrm{m}^{2}$ & $28.2 \pm 5.2$ \\
\hline Refractive error, $n(\%)$ & $38(40.9 \%)$ \\
\hline Intraocular pressure, $\mathrm{mmHg}$ & $12.7 \pm 3.0$ \\
\hline Diabetes mellitus, $n(\%){ }^{d}$ & $13(14.0 \%)$ \\
\hline History of cardiovascular disease, $n(\%)$ & $1(1.1 \%)$ \\
\hline Office hypertension, $n(\%)^{e}$ & $60(64.5 \%)$ \\
\hline On antihypertensive treatment, $n(\%)$ & $44(47.3 \%)$ \\
\hline Systolic/diastolic office blood pressure, $\mathrm{mmHg}^{f}$ & $141.0 \pm 22.8 / 76.2 \pm 8.3$ \\
\hline 24-h systolic/diastolic/mean arterial blood pressuref & $122.1 \pm 15.4 / 70.3 \pm 8.7 / 87.9 \pm 10.0$ \\
\hline Serum total cholesterol, $\mathrm{mg} / \mathrm{dL}^{g}$ & $220.7 \pm 47.7$ \\
\hline Serum high-density lipoprotein cholesterol, $\mathrm{mg} / \mathrm{dL}^{g}$ & $44.6 \pm 11.2$ \\
\hline Serum creatinine, $\mathrm{mg} / \mathrm{dL}^{g}$ & $0.90 \pm 0.26$ \\
\hline Blood glucose, $\mathrm{mg} / \mathrm{dL}{ }^{g}$ & $106.3 \pm 27.5$ \\
\hline $\mathrm{HbA} 1 \mathrm{c}, \%$ & $5.80 \pm 0.74$ \\
\hline \multicolumn{2}{|l|}{ Unless otherwise noted } \\
\hline \multicolumn{2}{|l|}{$b_{\text {IQR indicates interquartile range }}$} \\
\hline \multicolumn{2}{|l|}{$c_{\text {Use of smoking materials or alcohol on a daily basis }}$} \\
\hline \multicolumn{2}{|c|}{${ }^{d}$ Use of antidiabetic drugs or fasting blood glucose of $\geq 126 \mathrm{mg} / \mathrm{dL}(7.0 \mathrm{mmol} / \mathrm{L})$} \\
\hline \multicolumn{2}{|c|}{ An office blood pressure of $\geq 130 \mathrm{mmHg}$ systolic or $\geq 80 \mathrm{mmHg}$ diastolic or use of antihypertensive drugs } \\
\hline \multicolumn{2}{|c|}{$\begin{array}{l}f \\
\text { Office blood pressure was the average of five consecutive oscillometric readings at a single visit. The } 24 \text {-h blood pressure was measured by a } \\
\text { validated oscillometric device. }\end{array}$} \\
\hline
\end{tabular}

Hypertens Res. Author manuscript; available in PMC 2021 September 10. 


\section{을 \\ 골}

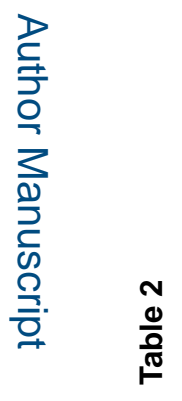

훙

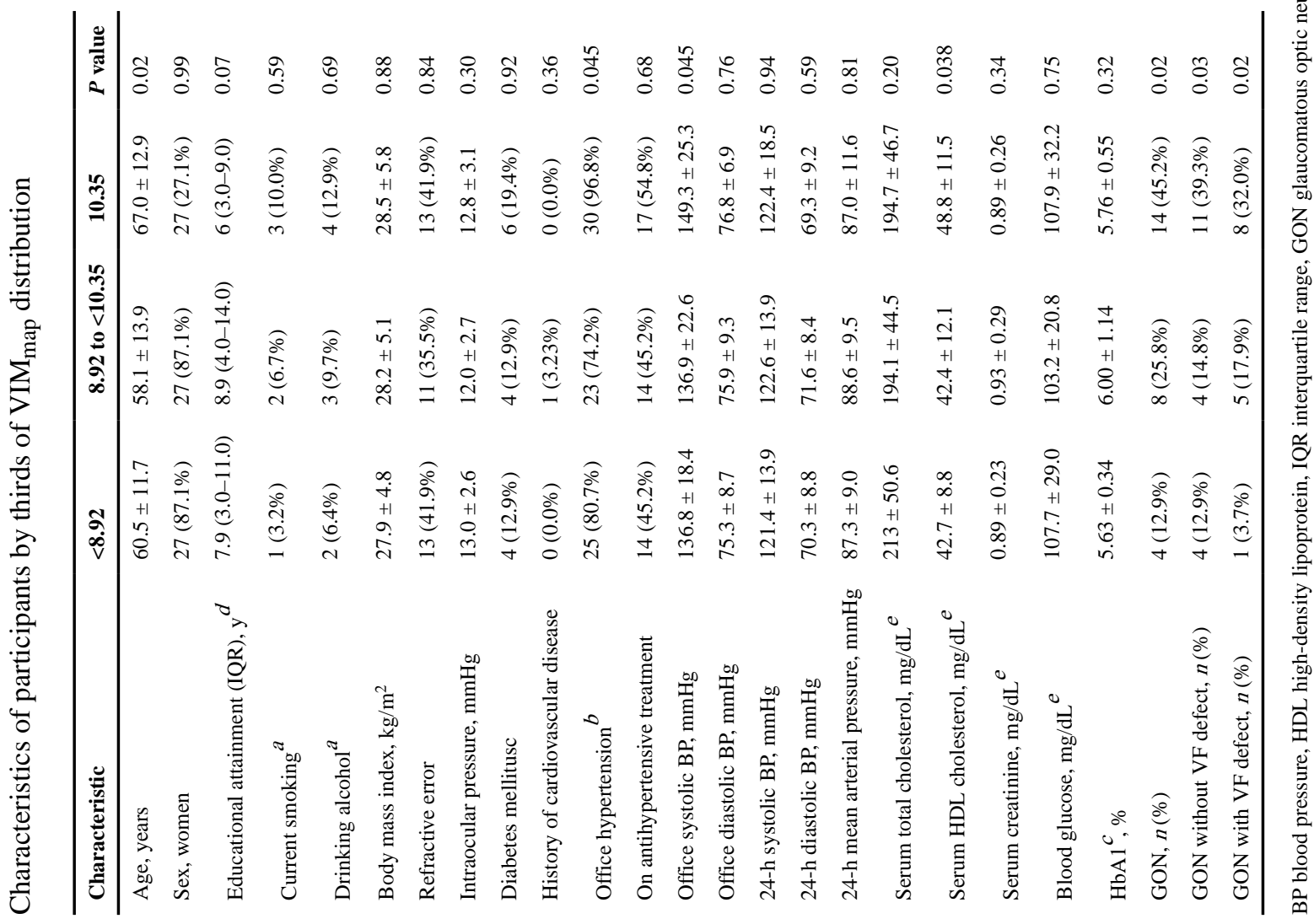

Hypertens Res. Author manuscript; available in PMC 2021 September 10. 

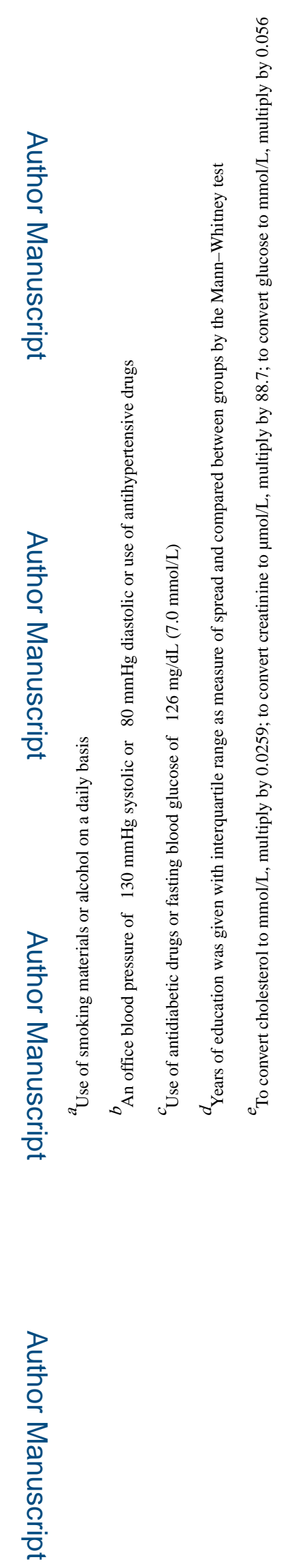

Hypertens Res. Author manuscript; available in PMC 2021 September 10. 